\title{
Reflectometric Measurement of Plasma Imaging and Applications
}

\author{
A. Mase ${ }^{a^{*}}$, N. Ito ${ }^{b}$, M. Oda $^{c}$, Y. Komada ${ }^{a}$, D. Nagae ${ }^{a}$, D. Zhang ${ }^{a}$, Y. Kogi $^{d}$, \\ S. Tobimatsu ${ }^{e}$, T. Maruyama ${ }^{e}$, H. Shimazu ${ }^{b}$, E. Sakata ${ }^{b}$, F. Sakaif, D. Kuwahara ${ }^{g}$, \\ T. Yoshinaga ${ }^{h}$, T. Tokuzawa ${ }^{h}$, Y. Nagayama ${ }^{h}$, K. Kawahata $^{h}$, S. Yamaguchi ${ }^{i}$, \\ S. Tsuji-lio ${ }^{g}$, C. W. Domier ${ }^{j}$, N. C. Luhmann, Jr. ${ }^{j}$, H. K. Park ${ }^{k}$, G. Yun ${ }^{k}$, W. Lee ${ }^{k}$, \\ S. Padhi', K. W. Kim ${ }^{m}$
}

${ }^{a}$ Art, Science and Technology Center for Cooperative Research, Kyushu University, Kasuga 816-8580, Japan

${ }^{b}$ Kyushu Hitachi Maxell, Ltd., Tagawa, Fukuoka 822-1296, Japan

${ }^{c}$ Miyazaki Prefecture Industrial Technology Center, Miyazaki 880-0303, Japan

${ }^{d}$ Department of Information Electronics, Fukuoka Institute of Technology, Fukuoka 811-0295, Japan

${ }^{e}$ Faculty of Medical Science, Kyushu University, Fukuoka 812-8582, Japan

${ }^{f}$ Sakura Tech Corporation, Kawasaki 213-0012, Japan

${ }^{g}$ Graduate School of Science and Engineering, Tokyo Institute of Technology, Tokyo 152-8550, Japan

${ }^{h}$ National Institute for Fusion Science, Toki 509-5292, Japan

${ }^{i}$ Faculty of Engineering Science, Kansai University, Suita 564-8680, Japan

${ }^{j}$ Department of Electrical and Computer Engineering, University of California, Davis, CA 95616, USA

${ }^{k}$ Department of Physics, Pohang University of Science and Technology, Pohang 790-784 Korea

${ }^{l}$ International Centre for Radio Astronomy Research, Curtin University, Bentley, WA, Australia

${ }^{m}$ School of Electronics Engineering, Kungpook National University, Daegue 702-701, Korea

E-mail: mase@astec.kyushu-u.ac.jp

ABSTRACT: Progress in microwave and millimeter-wave technologies has made possible advanced diagnostics for application to various fields, such as, plasma diagnostics, radio astronomy, alien substance detection, airborne and spaceborne imaging radars called as synthetic aperture radars, living body measurements. Transmission, reflection, scattering, and radiation processes of electromagnetic waves are utilized as diagnostic tools. In this report we focus on the reflectometric measurements and applications to biological signals (vital signal detection and breast cancer detection) as well as plasma diagnostics, specifically by use of imaging technique and ultra-wideband radar technique.

KEYWORDS: Microwave; Reflectometry; Imaging; Plasma Diagnostics, Biological Diagnostics

*Corresponding author 


\section{Contents}

1. Introduction 1

2. Reflectometric measurement of plasma imaging $\quad 2$

$\begin{array}{ll}2.1 \text { Experimental apparatus on LHD } & 2\end{array}$

2.1.1 Microwave/millimeter-wave system with quasi-optical transmission line 2

2.1.2 Notch filter and dichroic filter 4

$\begin{array}{ll}2.2 \text { LHD Experiment } & 7\end{array}$

3. Reflectometric measurement of biological signals $\quad 8$

$\begin{array}{ll}3.1 \text { Fluctuation measurement: vital signal detection } & 8\end{array}$

3.1.1 Heart beat measurement and application to stress evaluation $\quad 8$

3.1.2 Experimental apparatus: fixed frequency reflectometer $\quad 8$

3.1.3 Signal processing for reconstruction of HRV 9

3.1.4 Experimental results of HRV reconstruction and stress evaluation 10

3.1.5 Measurement of driver's vital signal 12

3.2 Microwave imaging: breast cancer detection 13

3.2.1 Motivation $\quad 13$

3.2.2 Numerical simulation $\quad 13$

3.2.3 Ultrashort-puluse reflectometer 14

$\begin{array}{ll}3.2 .4 \text { Experimental results } & 15\end{array}$

4. Conclusions 17

Acknowledgments

References

\section{Introduction}

Reflectometry has widely been used to measure plasma density profiles, density fluctuations, plasma rotation speed, and correlation lengths without perturbing the plasma [1-5]. When an electromagnetic wave is launched into the plasma, the wave is reflected at the corresponding cutoff layer. Due to high localization of measurements, noninvasive nature and low requirements to an access port window, reflectometry has become an ordinary diagnostics, and nowadays it is utilized on a number of magnetic fusion devices.

Within the last two decades, plasma fluctuations are of much interest and application of reflectometry to fluctuations measurements is under investigation. In microwave reflectometry, the WKB approximation $[6,7]$ is often used for interpretation of the received signal. According to this approximation, plasma fluctuations are supposed to predominantly affect phase of a probing signal and do not change its amplitude (phase-screen model). Therefore, near the cutoff region the shape of signal wavefronts corresponds to the shape of fluctuations [4]. If the 
amplitude of fluctuations is small or the width of their wavenumber spectrum is narrow, the microwaves bear phase information on a large distance and interpretation of the signal is straightforward. Otherwise, interference of the scattered wavefronts destructs the shape of the phase already close to the cutoff position making difficult extraction of the information from the reflectometer signal. One of the possible ways to solve the problem, which is used in microwave imaging reflectometry (MIR) [8-10], is to use large aperture optics to restore the wavefronts at the receiver position. The MIR concept is valid until the cutoff can be considered as the phasescreen, and the optics are able to build an image of the cutoff.

Microwave/Millimeter-wave reflectometry has also become of importance in various applications such as remote sensing, biomedical and environmental measurements as well as plasma diagnostics. In this report we focus on the reflectometric measurements applied to biological signals, specifically by use of imaging technique and ultra-wide band radar technique. The paper is divided into three sections. The development of a millimeterwave imaging system applied to the LHD plasma is described in Sec. 2. The diagnostic system has also applied to other applications as nonperturbing methods. Section 3 denotes the brief description of the applications to biomedical measurements, and is followed by conclusion.

\section{Reflectometric measurement of plasma imaging}

\subsection{Experimental Apparatus on LHD}

\subsubsection{Microwave/millimeter-wave system with quasi-optical transmission line}

A combined system of MIR and ECEI (electron cyclotron emission imaging) as shown in Fig. 1 has been applied to the Large Helical Device (LHD) [11-13]. The MIR system uses 4 different frequency sources in order to obtain the information at 4 radial positions. The sources are obtained as follows [13]. The 4 frequencies at 750, 983, 1183 and $1450 \mathrm{MHz}$ from the crystal oscillators are separately up-converted with the $18.33 \mathrm{MHz}$ from the crystal oscillator. These 4 frequencies are mixed with the carrier wave frequency at $9.3 \mathrm{GHz}$ from a voltage-controlledoscillator (VCO). Upper side-band frequency components at 10.068, 10.301, 10.501 and 10.768 $\mathrm{GHz}$ are utilized. The bandpass filters (BPFs) drop unnecessary lower side-band components to lower than $-40 \mathrm{~dB}$. The 4 frequencies are separately sextupled, and then combined by directional couplers. The output signal with frequencies of $60.410,61.808,63.008$ and $64.610 \mathrm{GHz}$ is then amplified and irradiated to a target plasma as a probing beam.

In the early stage of experiment, reflected waves from the cutoff surfaces corresponding to each frequency are focused via the imaging optics onto an aperture of 2-D horn antenna array (HAA) with mixers [12]. The array consists of 35 small horns aligned in toroidally 5 by poloidally 7 channels. The received wave is mixed with the first-LO signal at $55.800 \mathrm{GHz}$ by Schottky diode mixers. The frequency is obtained by sextupling $9.300 \mathrm{GHz}$ from a VCO. The down-converted first-IF signal (RF-LO) consists of 4 frequency components at 4.610, 6.008, 7.208 and $8.810 \mathrm{GHz}$. These first-IF frequency components are separated by a BPF bank fabricated by microwave integrated circuit (MIC) technology [14]. The separated first-IF signals are down-converted again by mixing with 4 second-LO signals $(4.500,5.898,7.098$ and 8.700 $\mathrm{GHz}$ ). The second-IF signals all at $110 \mathrm{MHz}$ are finally obtained for the amplitude and phase detection by the power detector and the quadrature-demodulator. Recently, a 2-D horn-antenna mixer array (HMA) is developed instead of HAA, which consists of arrayed quasi-optical antenna-mixers covered with pyramidal horn apertures [15].

The ellipsoidal or hyperboloidal surfaces of the curved aluminum alloy mirrors were 
determined for simultaneous focusing of the above three wave components (the illuminating waves, the reflected waves, and the first LO wave). The focusing optics of the reflected waves on the HMA aperture and the illuminating optics for probe waves were designed first to match the existing movable main mirror. The LO wave transmission optics was matched to the previously designed mirror so that the LO beam covers the whole aperture area with a $100 \mathrm{~mm} \times 100 \mathrm{~mm}$ rectangle to supply enough power for down-conversion $(-16 \mathrm{dBm})$ to the mixer diodes. The optics is designed by using the 2-D finite-difference time-domain (FDTD) method [16]. Since the numerical solutions of the Maxwell equations are directly computed in the FDTD calculation, the effect of finite wavelength can be intrinsically simulated. An adjustable ellipsoidal mirror is installed in the vacuum chamber in order to control the illumination angle. The mirror angles are remotely controlled by non-magnetic actuators with ultrasonic motors as shown in Fig. 1. Separation between the illumination and the detection optics reduces the back ground noise due to the stray wave. Note that the adjustment of the mirror angle is extremely important for the LHD experiment, since the optimum angle can be lost even in 1 degree change.
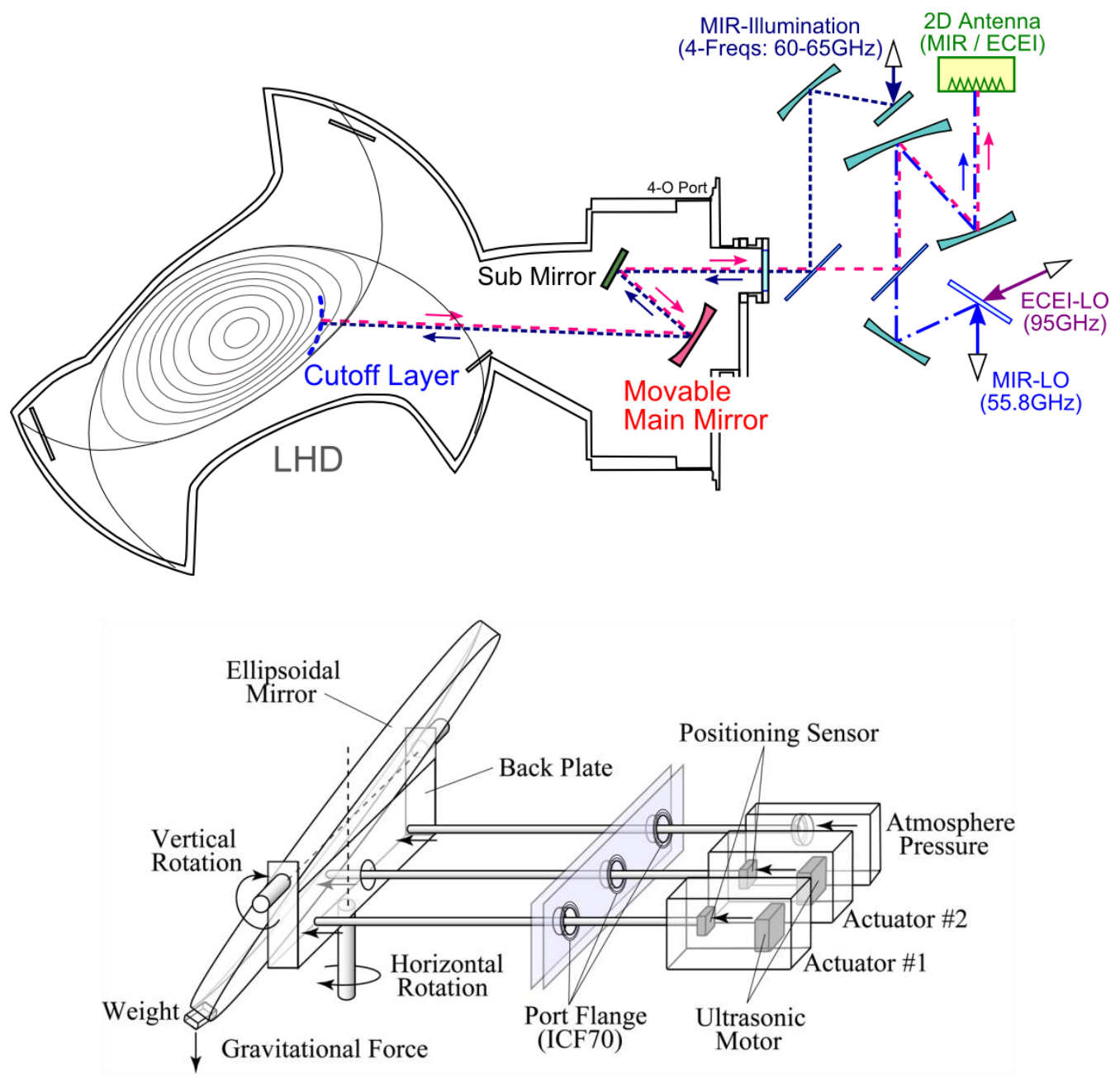

Fig. 1. Microwave imaging system on LHD: microwave system and optics (upper), remote control system of the focusing mirror (lower). 


\subsubsection{Notch filter and dichroic filter}

Protection of the microwave/millimeter-wave diagnostics from stray radiation of electron cyclotron resonance heating $(\mathrm{ECRH})$ power has been one of the critical issues on magnetic confinement systems. Ranges of the problems are as follows: i) signal corruption (power loading of microwave mixers), ii) destruction of sensitive detectors and ex-vessel microwave components, iii) damage to in-vessel components due to thermal effect. A band-stop filter (notch filter) can be one of the most effective solutions to avoid these problems [17]. Typical ECRH heating frequencies for fusion plasma devices are 77, 84, 110, 140, and $170 \mathrm{GHz}$. For the LHD-MIR/ECEI experiment, a frequency selective surface (FSS) notch filter is fabricated and applied.

The FSS consists of an array of periodic metallic patches on a dielectric substrate or a periodically perforated conducting sheet [18]. A square loop FSS filter structure was selected for the present experiment. The geometry is shown in Fig. 2. The equivalent circuit of the loop corresponds to LC resonant circuit. From the equivalent circuit, it can be seen that the wider conducting line yields a smaller resistance. The unit cell size determines the frequency where diffraction causes grating lobes and also determines the resonant frequency by modifying the mutual capacitance.

As the first requirement, a FSS with notch frequency of $77 \mathrm{GHz}$ has to be designed and fabricated. The dielectric constant and substrate thickness can also affect the transmission performance. A $0.254 \mathrm{~mm}$ thick substrate (Nippon Pillar Packing Co. Ltd.: NPC-F220A) is chosen. The parameters of the substrate are as follows: copper thickness $0.009 \mathrm{~mm}$, and dielectric constant and loss tangent measured at $10 \mathrm{GHz}$ are 2.2 and 0.0007 , respectively. In order to optimize the center frequency, it is necessary to determine the unit size and geometry of the loop. We have fabricated FSS filters with various sizes of conductor line width. Figure 3 shows the observed center (notch) frequency as a function of the line width. It is seen that the notch frequency almost linearly depends on the line width. Thus, we can fix the line width to $0.143 \mathrm{~mm}$ for the present requirement.

The characterization of the notch filter $\left(S_{21}\right.$ vs. frequency, where $S_{21}$ is the transmission coefficient) applied to the LHD experiment is shown in Fig. 4(a). The rejection of larger than 35 $\mathrm{dB}$ is obtained at the notch frequency of $77 \mathrm{GHz}$. By integrating 2-3 filters, we can obtain large rejection ratio (> $60 \mathrm{~dB}$ ) as shown in Fig. 4(b).

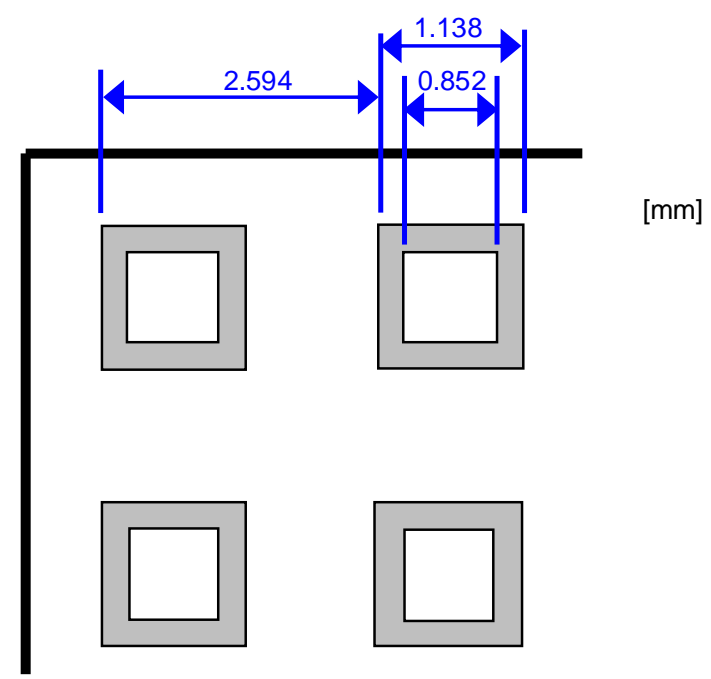

Fig. 2. Geometry of square loop FSS. 


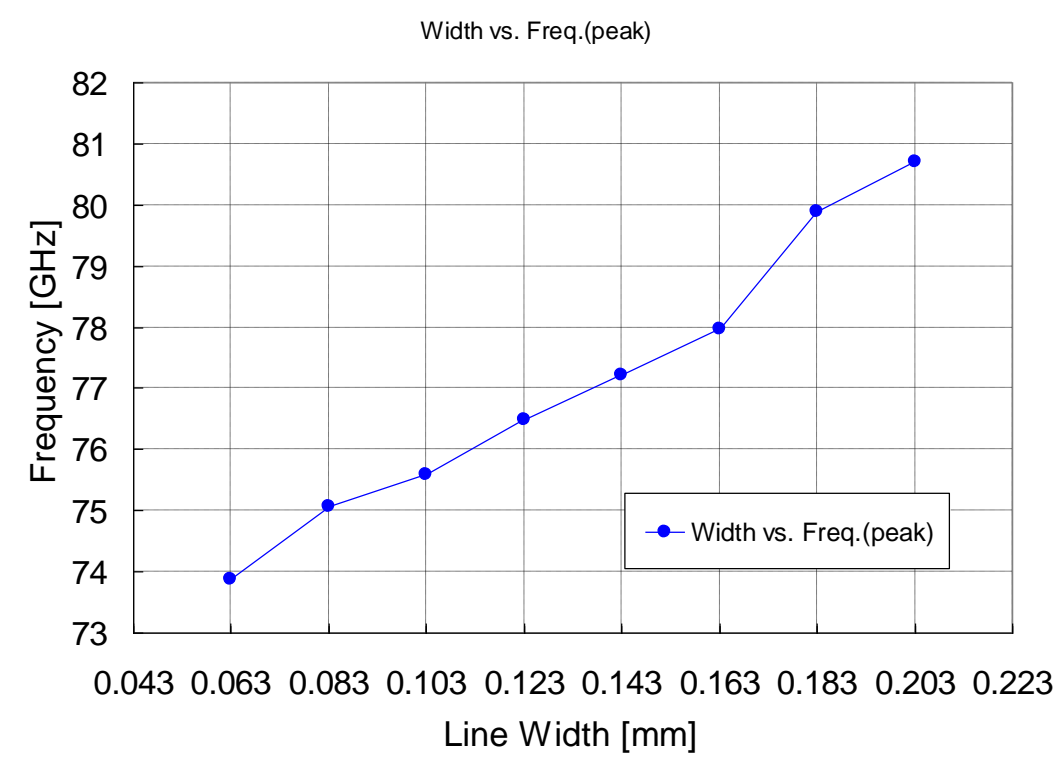

Fig. 3. Notch frequency as a function of conductor line width.

(a)

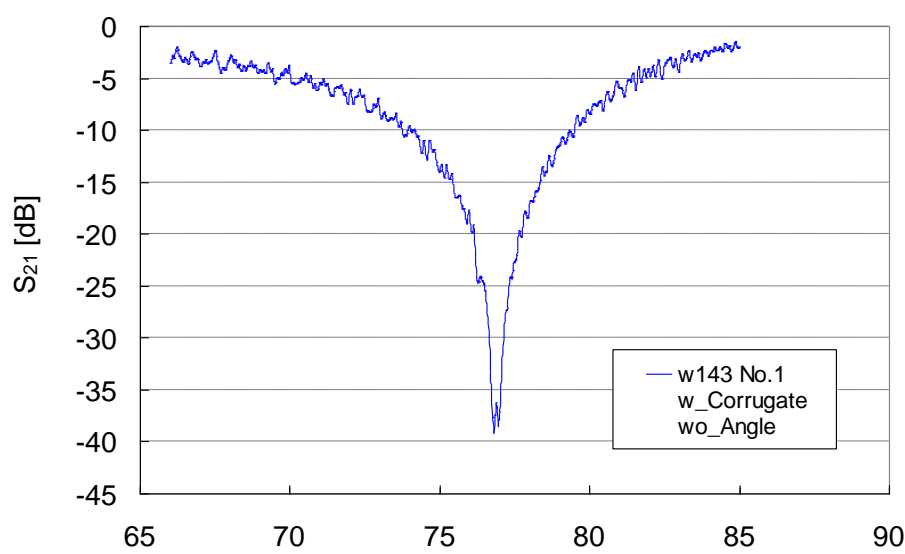

(b)

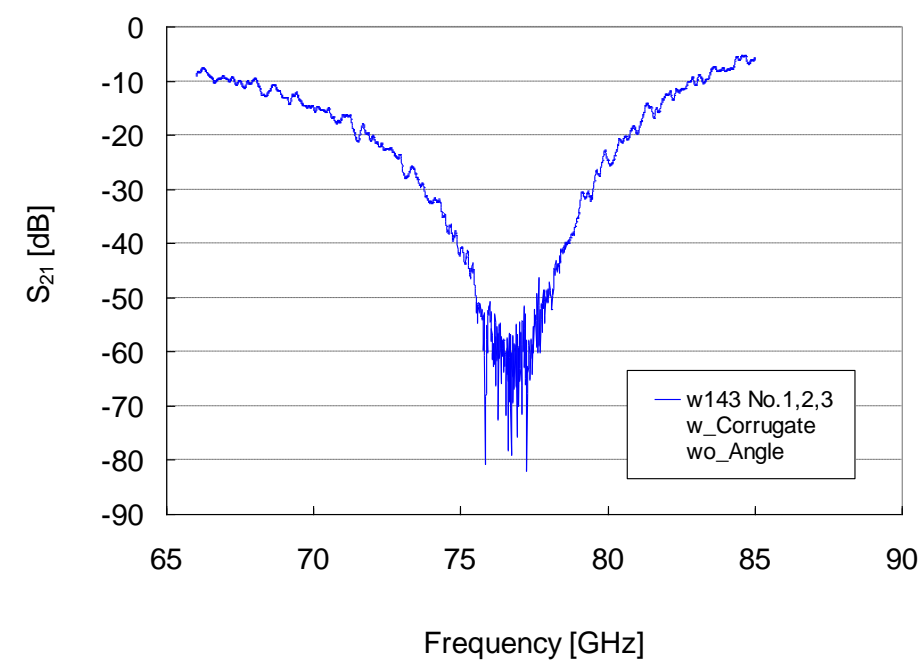

Fig. 4. Transmission coefficient vs. frequency: (a) single, (b) triple. 
Metallic sheets with perforated with circular holes have been widely used for reflectors and filters. This type of filter, called as a dichroic filter, can also be utilized as a beam splitter to divide two different frequency components as well as high-pass filter to prevent the low frequency ECRH stray radiation. The transmission coefficient of a dichroic plate with circular holes located equilateral triangular lattice is theoretically calculated in Ref. 19.

Figure 5 shows the transmission coefficient $\left(S_{21}\right)$ of the dichroic filter made of $6.35 \mathrm{~mm}$ thick aluminum with hole sizes of $1.55 \mathrm{~mm}$ diameter and $2.34 \mathrm{~mm}$ spacing shown in the righthand side, which is in good agreement with the calculated one. The cutoff frequency is 113.3 GHz. The transmission characteristics can be adjusted when the spacing between the holes is changed as shown in Fig. 6 . When the cutoff frequency of $200 \mathrm{GHz}$ or more is needed, the hole diameter becomes less than $1 \mathrm{~mm}$. Machining of the dichroic filter of such diameter is quite difficult. We have fabricated two same dichroic plates with half thickness. By piling up them and adjusting the hole position, we have obtained good performance of the filter.

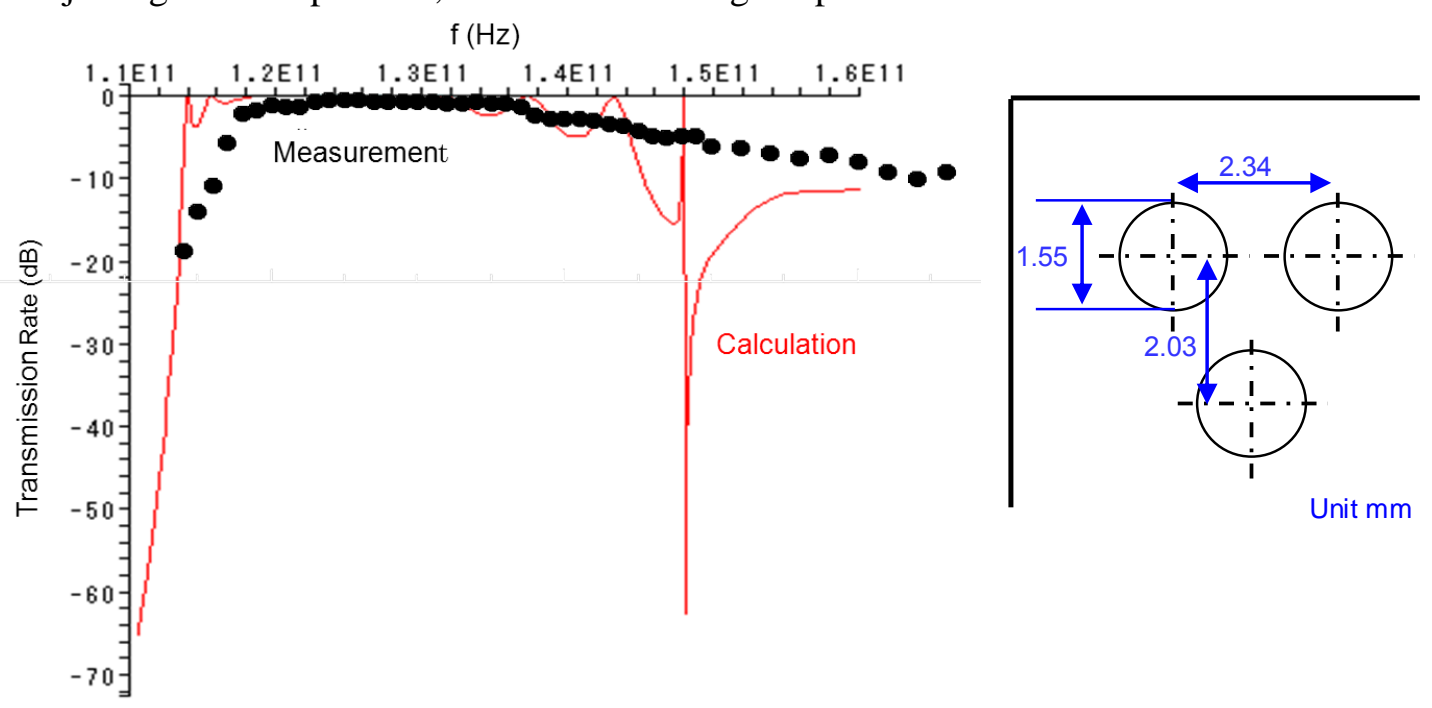

Fig. 5. Measured and calculated transmission coefficient of the dichroic plate.

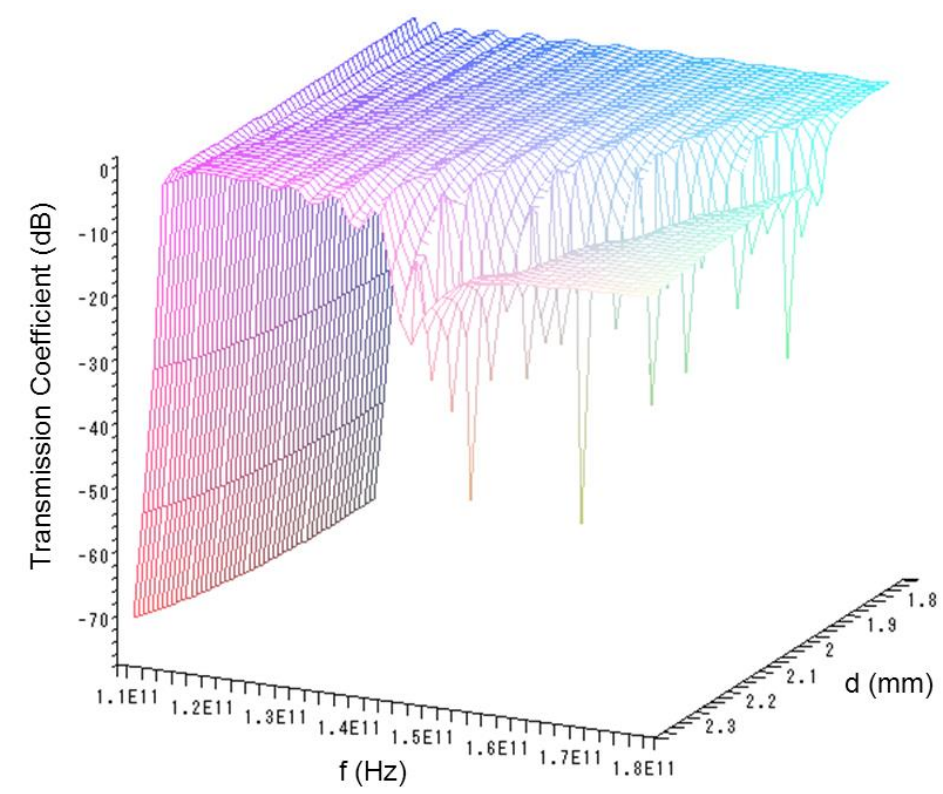

Fig. 6. Transmission coefficient vs. frequency for various values of spacing d. 


\subsection{LHD experiment}

Figure 7 shows typical waveforms of the MIR signals (amplitude and quadrature phase) together with a magnetic probe signal [13]. The MIR signals in this discharge appear to have a certain $\mathrm{S} / \mathrm{N}$ ratios. The fluctuating signals in both amplitude and phases are excited during the period of finite electron density. This suggests that the present MIR system can achieve those fluctuation signals from the plasma itself rather than from the electron-cyclotron (EC) heating pulses or the neutral-beam (NB) injections. The fast Fourier transform (FFT) spectra of the MIR signals accompany equally-spaced higher order harmonics and are quite similar to each other as shown in Fig. 7. The components with the fundamental and the second harmonic frequency at several $\mathrm{kHz}$ can be found also in the magnetic fluctuation's spectrum. This also supports that the MIR system is indeed observing the plasma. Meanwhile, the higher order harmonics can only be seen in the MIR signals, and there are some frequency components which can be found only in the magnetic probe signal (e.g. $f=7-8 \mathrm{kHz}$ and $13-14 \mathrm{kHz}$ during $t=4.0-4.2 \mathrm{~s}$ ). Moreover, the perturbation envelopes from these two diagnostics scheme are also quite different. The difference between these two diagnostics systems would be originated from the difference of those inherent characteristics. As an example, MIR is a local diagnostics in the vicinity of the cutoff surface while the magnetics reflects information from a wide area in the plasmas. The frequency components appearing only in the magnetic signal might be arising from the inner region of the cutoff surfaces observed on MIR. The mode is destabilized by both high-energy ion and pressure gradient at the rational surface. The first result of 3-D image of the MHD instability shows that a localized fluctuation burst is observed on the edge plasma in LHD [20].

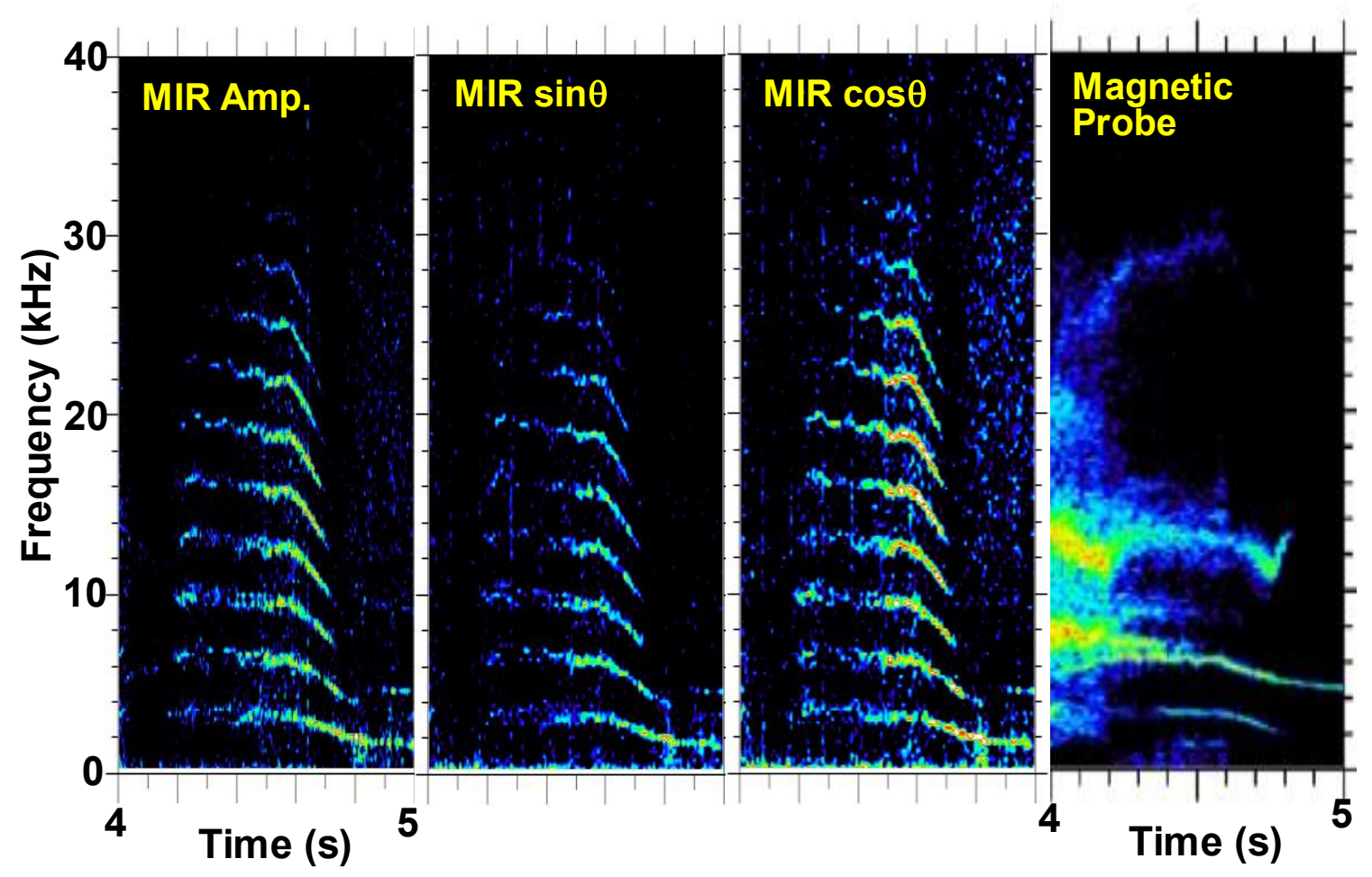

Fig. 7. Experimental result on LHD: Time history of FFT spectra of MIR signal and magnetic probe signal. 


\section{Reflectometric measurement of biological signals}

\subsection{Fluctuation measurement: vital signal detection}

\subsubsection{Heart beat measurement and application to stress evaluation}

It is said recently that a large percentage of population suffers from chronic disease such as asthma, diabetes or heart diseases. The healthcare and monitoring become of growing importance. Heartbeat signal is a very useful parameter for such monitoring. Several methods have been developed except conventional electrocardiogram (ECG), such as, piezo-electric sensor, ultrasonic sensor, and microwave reflectometry sensor. Microwave reflectometer measures remotely the human skin and internal organs movements when breathing and heartbeat are present rather than the electrical impulses measured by ECG [21-24].

The heartbeat interval is constantly fluctuating by autonomous nerve activity comprised of sympathetic and parasympathetic nerve activity. The time variation can be regarded as the shortterm time variation of the heartbeat frequency, so called, the heart rate variability (HRV). The HRV is obtained by interpreting the R-R intervals in an ECG data. In the frequency spectrum of the HRV, the parasympathetic nerve activity appears in the spectral region of $0.15-0.45 \mathrm{~Hz}$ (so called, HF region), and both sympathetic and parasympathetic nerve activity appear in the region of $0.03-0.15 \mathrm{~Hz}$ (LF region) $[25,26]$. The peak ratio or area ratio of the power spectrum in the LF component to the HF component is used to evaluate the stress. The sympathetic nerve activity increases in a stressful state and the parasympathetic nerve activity increases in a relaxed state. The ratio LF/HF in a stressful state is larger than in a relaxed state [27, 28].

It is noted that the evaluation of the stress by ECG has long been suggested as a reliable means to estimate mental stress. However, the stress evaluation method using the ECG seems to be unsuitable as a long-time monitoring subject, as a several electrodes are attached directly to the human body to acquire the ECG data. Specifically, there is some concern about the practical application of the ECG system to sick person and elderly person. Consequently, we propose using a non-contacting and non-invasive way to predict the evaluation of stress.

\subsubsection{Experimental apparatus: fixed-frequency reflectometer}

A heterodyne-type reflectometer as shown in Fig. 8 is applied to the present experiment. A microwave oscillator with frequency of $10 \mathrm{GHz}$ is used as an incident wave and a local oscillator (LO) wave. One of the waves passing through a directional coupler is shifted by the signal from a VHF oscillator with frequency of $110 \mathrm{MHz}$ using a single-sideband up-converter, which is irradiated onto the vicinity of human heart or the path of an artery in the thigh via a 16element patch antenna array. The reflected wave from the skin and the surface of the heart is picked up by a single patch antenna, and down-converted to an intermediate frequency (IF) signal of $110 \mathrm{MHz}$. An automatic gain control (AGC) amplifier with frequency range of 100$200 \mathrm{MHz}$ is utilized to control the reflected wave in both static and non-static environment. The quadrature phase detector provides two components in the mixer output, $E_{r} E_{l} \cos \Delta \phi$ and $E_{r} E_{l} \sin \Delta \phi$, where $E_{r}$ and $E_{l}$ is the amplitude of the reflected wave and the local oscillator wave respectively, and $\Delta \phi$ is the phase difference between the reflected wave and the local oscillator wave. We can distinguish the amplitude and the phase difference of the reflectometer signals by detecting the above two components. The AC components of the phase difference include the periodic movement of the reflection layer due to the heart beat as well as random movement of the human body. The body movement due to respiration is a centimeter or so, and the motion due to heartbeat is typically only a fraction of a millimeter. It is known that a microwave 


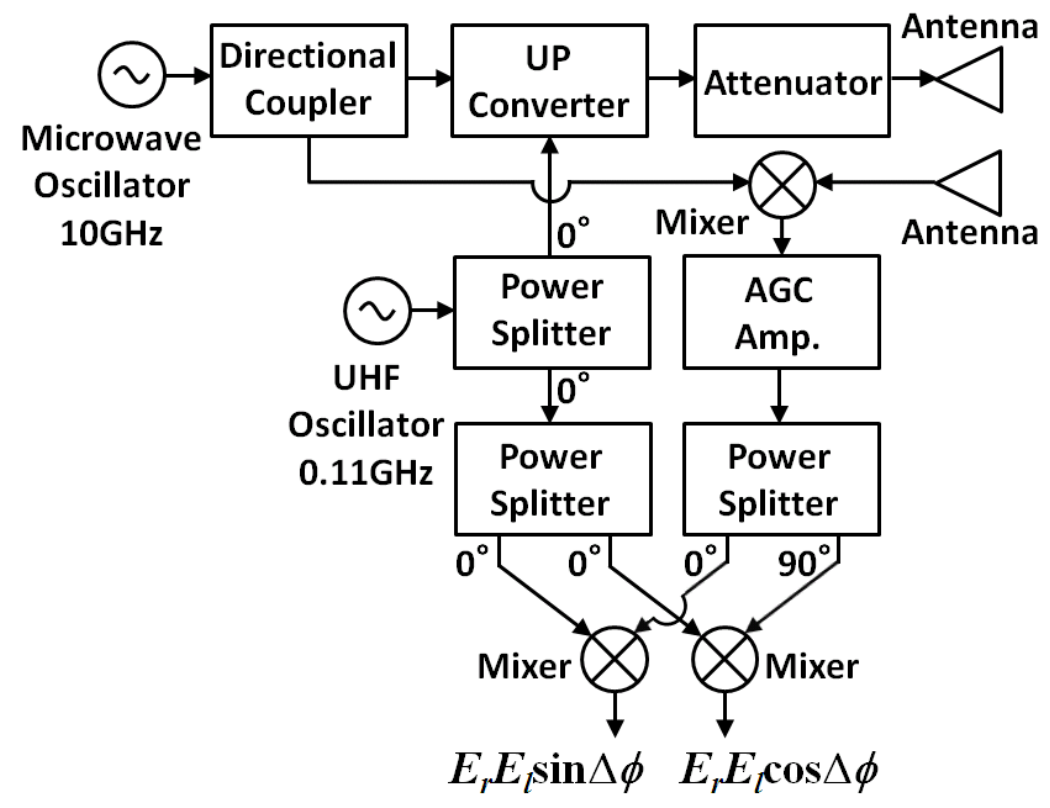

Fig. 8. Schematic of the microwave reflectometer for vital signal detection.

reflectometer typically has a phase resolution of 1/200 fringe which corresponds to a spatial resolution of $\lambda / 400$, where $\lambda$ is the incident wavelength. Thus, the resolution becomes $75 \mu \mathrm{m}$. Therefore, the body movement due to the respiration and heartbeat could be detected easily.

To extract the heartbeat signal from the measured signal, for example, the respiration signal is removed by a high-pass filter with a cutoff frequency of $0.7 \mathrm{~Hz}$. The spurious component of the reflected wave due to random movement of the human subject is difficult to be removed, since it has a wide frequency spectrum. Additionally, the heartbeat signal is very small and has low signal-to-noise ratio, because the cardiac movement appears on the body surface through the bone and the connective tissue. Thus, the peak positions in the heartbeat signal obtained by a microwave reflectometer are not as clear as those observed in the ECG signals, which makes it difficult to infer the heart rate correctly by interpreting the peak intervals of the microwave measurement signal without any data processing.

\subsubsection{Signal processing for reconstruction of $\mathrm{HRV}$}

We have proposed the cross-correlation technique, which is a method reconstructing the HRV from the cross-correlation function between a measurement signal and a template signal obtained by averaging the periodic waveform in the measured signal [29]. However, it is difficult to reconstruct the HRV in real time by the cross-correlation technique. This defies the detection of stress state timely. In this section, the time variation of the heartbeat frequency is evaluated by applying the maximum entropy method (MEM) repeatedly. The time window is shifted step by step along the temporal axis. Then, the HRV can be obtained since the value of the heartbeat interval is calculated by the inverse of the heartbeat frequency. The MEM is an effective application for estimating the frequency spectrum at a short data window.

In the MEM, the model order is generally unknown. If the model order is selected lower, the estimated spectrum is smoothed and the spectrum peak which should be found in a normal situation does not often appear. On the other hand, if the model order is selected higher, a lot of 
spurious peaks appear finely in the estimated spectrum. The optimum selection of the model order is of importance in the MEM [30,31]. We have selected the optimum model order as 870 from the empirically-based experiences.

The heartbeat frequency is estimated by finding the largest peak in the frequency range of $0.7-1.55 \mathrm{~Hz}$ (42-98 bmp) after calculating the power spectrum of some data windows by the MEM. Here, the frequency $0.7-1.55 \mathrm{~Hz}$ covers sufficiently the range of heartbeat frequency for a seated human subject at rest. It is said that the heart rate for a seated human subject is typically in the range of 45-90 bmp, i.e., $0.75-1.5 \mathrm{~Hz}$. If the analyzing data window is selected long, time response of the heartbeat intervals becomes sluggish in the HRV while the reliability of the estimation enlarges. This could exert a bad influence upon the estimation of the LF/HF. In this paper, $2.5 \mathrm{sec}$ is selected as the optimum length of the data window for the MEM analysis from the empirically-based experiences.

In addition, $0.25 \mathrm{sec}$ is selected as the shift quantity of the data window. It is equal to the interpolated value of the HRV reconstructed by the ECG. Unlike the non-stationary spectrum analysis techniques, the MEM is highly regarded as a reliable stationary spectrum analysis technique where the consistency of the signal in the data window is achieved. As the correspondence relationship between the data windows is ignored, some spectrum estimation errors due to the selected data window appear as the fluctuation with a period of $0.25 \mathrm{sec}$ which is equivalent to $4 \mathrm{~Hz}$ in frequency in the HRV signal. This is not a problem, however, for the evaluation of the stress, because the spectrum estimation error between data windows is very small and its fluctuation does not extend over the frequency region of the LF and the HF. For these reasons, the time variation of the heartbeat frequency is estimated by repeatedly applying the MEM at the $2.5 \mathrm{sec}$ data window which is then shifted by $0.25 \mathrm{sec}$ along the temporal axis.

\subsubsection{Experimental results of HRV reconstruction and stress evaluation}

The heartbeat measurement by a microwave reflectometer is applied to the person who is in a static or non-static environment. In a non-static environment, the microwave reflectometer signal includes various noise components due to random movements of the body surface, thus making it harder to detect the heartbeat interval. Figure 9 shows an example of the measurement with such noise components applied to a person sitting in a driver's seat with engine vibration together with the ECG data. Note that the peak (R-R) interval is easy to obtain from the ECG signal, however, it is not clear in the microwave signal.

(a)

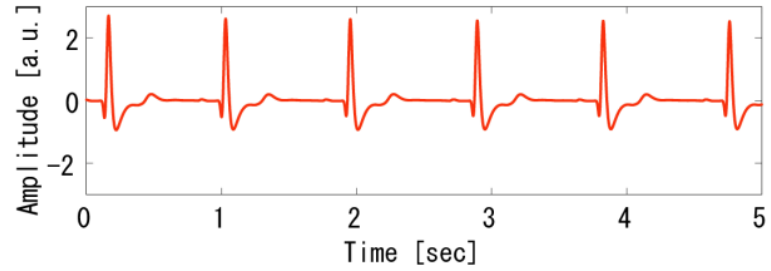

(b)

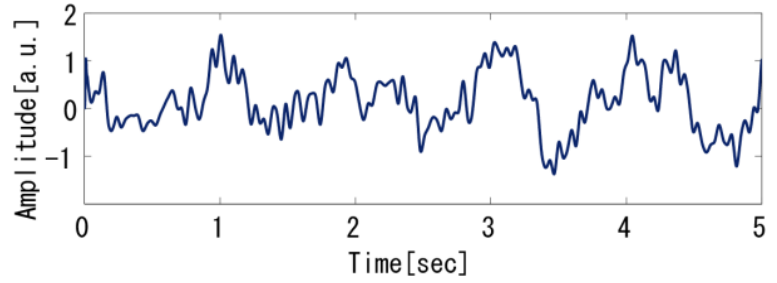

Fig. 9. Time evolution of (a) the ECG signal and (b) the microwave reflectometer signal. 
In Fig. 10 is shown the frequency spectra of the HRV reconstructed by two methods, the cross-correlation and the MEM, together with those obtained from the ECG signals. In this case, the calculation of the cross-correlation was repeated three times to make the peak intervals of the waveform clear [29]. The spectral shapes of the HRV using the third cross-correlation function and the MEM technique is nearly equal to that by the ECG and their LF/HFs lie in the range of $\pm 30 \%$ compared to that obtained by the ECG. Those results show that the evaluation of the stress can be performed by applying two proposed methods to microwave reflectometer signals similar to the ECGs even in non-static environment.

Figure 11 shows an example of the measurements. The stress evaluation methods are applied to a person in both relaxed state and stressful states for long time of $900 \mathrm{sec}$. The flash mental calculation is used to put stress on a person where the figures are displayed continuously on a monitor before the subject's head [32,33]. During the time interval of 360-540 sec, the stress is put on a person. Other interval 0-360 sec and 360-900 sec the person is in a relaxed state. The algorithm based on the MEM made possible to reconstruct the HRV correctly and automatically from low-S/N microwave reflectometer signals. However, as far as we use FFT to obtain the frequency spectrum of the HRV data, we need much time to process, such as $180 \mathrm{sec}$. Therefore, we have applied wavelet transform for short time processing. The time-frequency spectrum of the MEM based HRV data obtained is analyzed by wavelet transform. Note that the

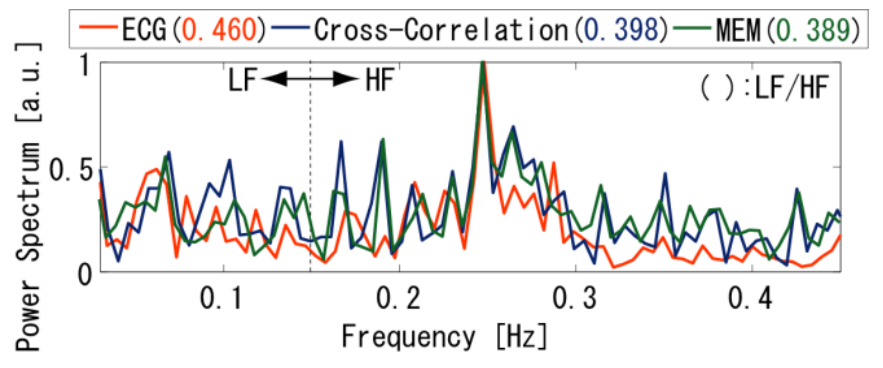

Fig. 10. FFT spectra of the HRV reconstructed by the MEM, the cross-correlation technique and the ECG signal.

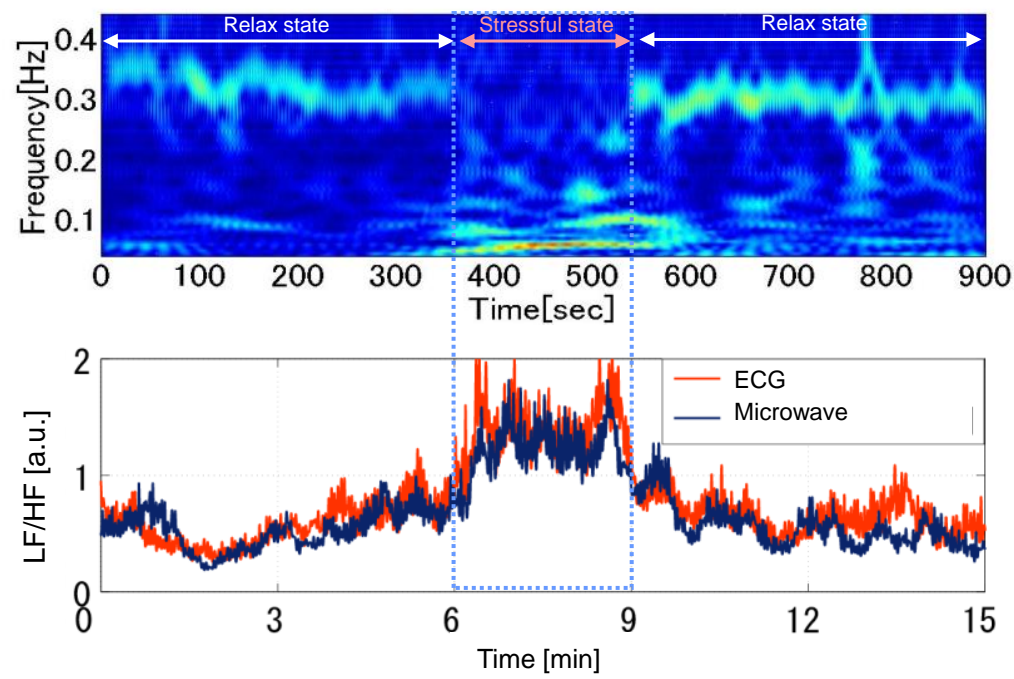

Fig. 11. Wavelet spectrum of the HRV (upper trace); The LF/HF value as a function of time (lower trace). 
HF components dominate at the relax state, while the LF components dominate at the stressful state. The time change rates of the LF/HF obtained from the microwave measurement are in good agreement with that obtained from the ECG, since the frequency spectra of the heart rate variability obtained by applying the MEM to the microwave measurement were nearly conformable to the data obtained by the ECG. The results show the possibility of long-term and real-time stress monitoring.

\subsubsection{Measurement of driver's vital signal}

Here we describe the measurement of driver's heartbeat using a microwave reflectometer. It is known that driver's physiological state is reflected by vital signals, such as heartbeat, respiration, blood pressure, body temperature and etc. Specifically, the heart rate variability (HRV) obtained from heartbeat measurement can be used for stress evaluation, which is considered to be useful to detect drowsy driving and drinking driving. In the present experiment the driver's heartbeat is measured by a heterodyne-type I-Q detection system with an AGC amplifier (Fig. 8) which actualizes high accuracy of the measurement.

In the driving condition, the microwave measurement signal includes random and large body movements due to road noise and steering wheel control. The movements often have similar periodicity to the heartbeat rhythm. It is therefore difficult not only to estimate the stress but even to measure the heartbeat signal. We have succeeded to reduce the noise component and extract only the heartbeat signal by calculating a cross-correlation function between two measurement signals obtained from two microwave reflectometers. Figure 12 (a) and (b) show the wavelet spectra of the signal obtained from each reflectometer, and Figure 12(c) shows the cross-spectrum between two signals. It is noted that the signal-to-noise ratio of the heartbeat spectrum is improved.

Figure 13 shows a wavelet spectrum of the reflectometer signal and the time variations of heartbeat obtained from two reflectometer signals and by the ECG. The success rate of the heartbeat measurement by the microwave reflectometer is $81 \%$. The success rate means the ratio of the measurement time to the term, in which the error of time variations of heart rate is kept within $\pm 10 \%$. Present microwave refelctometer system seems to have enough performance for the measurement of heartbeat during driving condition.

(a)

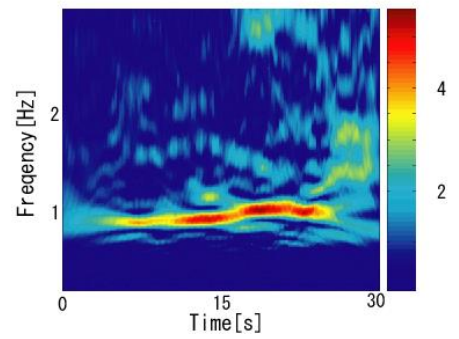

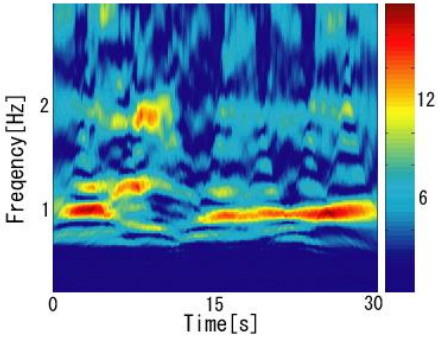

(c)

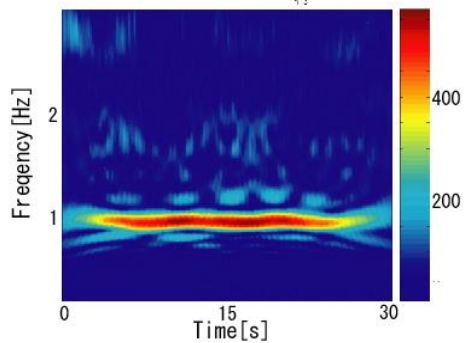

(b)

Fig. 12. Wavelet spectrum of each reflectometer signal (a, b) and cross-correlation spectrum (c). 

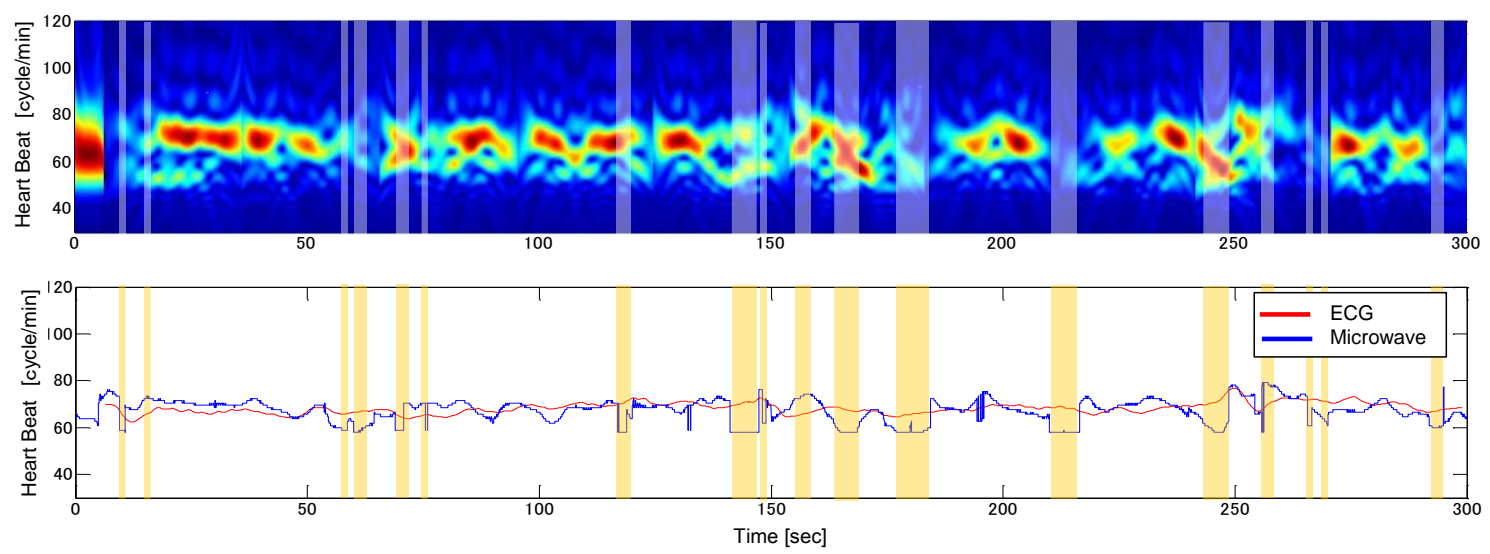

Fig. 13. Wavelet spectrum of the reflectometer signal (upper trace) and time variation of the peak values of heart beat during driving for two methods (ECG and microwave) (lower trace).

\subsection{Microwave imaging for breast cancer detection}

\subsubsection{Motivation}

Breast cancer incidence and mortality increase every year. It becomes important to detect earlystage tumors for healing. X-ray mammography is currently used for breast cancer detection, however, there is a limit in detection sensitivity, since the contrast of dielectric constant between malignant and normal breast tissues is small in the X-ray frequencies comparing to that in microwave frequencies. In microwave region, the contrast is said to be as large as 10:1 [34], and the attenuation of electromagnetic wave in normal breast tissue is comparatively small. Also breast compression required for clear image with X-ray mammography often causes a pain to patients. A burden to patients will be reduced by applying an ultrashort-pulse radar (USPR) to breast imaging since it can perform contactless inspection. Therefore the breast imaging with the USPR has potential to be used concomitantly with, or instead of X-ray mammography.

\subsubsection{Numerical simulation}

In numerical simulations, we solve Maxwell's equations describing the propagation of electromagnetic wave and its scattering and reflection by an object. The numerical scheme is based on the finite-difference time-domain (FDTD) model, and the simulations are performed in two-dimensional space [35].

The optical properties of a material are generally characterized by the complex permittivity. A foreign object having a permittivity different from an object to be inspected causes wave reflection and scattering. Breast has rather simple structure and lower dielectric constant and loss tangent than the other part of human body. In addition, the dielectric properties of breast tumors are much higher than the normal breast tissue. We assume that the dielectric property of normal breast tissue is $\varepsilon_{r}=9, \sigma=0.4 \mathrm{~S} / \mathrm{m}$ and that of the malignant breast tissue is $\varepsilon_{r}=50, \square \sigma=4 \mathrm{~S} / \mathrm{m}$ [36]. A Gaussian-shape pulse with a 65 ps full-width halfmaximum (FWHM) is irradiated to the breast through an antenna located at the position of 10 $\mathrm{cm}$ apart from the breast surface. The antenna is rotated every 10 degree around the breast from 
0 to 180 degrees. The reflected waves from the skin and the tumor are received by the identical antenna.

The reconstruction method of image proposed to the present breast cancer detection named as confocal microwave imaging (CMI) bases on a synthetic aperture radar (SAR) [37-39]. In our FDTD simulation, the numbers of antenna position correspond to $M=19$. The calculation process is repeated at each antenna position. Although the incident wave and the reflected wave from the breast skin are much larger than that from the tumor, it is not difficult to distinguish those waves due to the difference of the time-of-flight. Therefore, the spurious signals can be removed.

To reconstruct the image, a point is focused. First, the distances between the focal point and each antenna position are determined and converted to the time delays. The reflected waves obtained by each antenna are summed up, and the square of this sum is assigned to the pixel value at the focal point. The intensity $(I)$ of the pixel is assigned as

$$
\begin{aligned}
& I(\rho)=\left[\sum_{m=1}^{M} B_{m}\left(\tau_{m}(\rho)\right)\right]^{2} \\
& \tau_{m}(\rho)=2\left|r-\rho_{m}\right| / v \Delta t
\end{aligned}
$$

where $B_{m}$ is the backscattered waveform at the $m$ th antenna located at $r_{m}$, and $\tau_{m}(r)$ is the time delay from the $m$ th antenna to the synthetic focal point at $r$. Here, the propagation velocity inside the breast is calculated by using the assumed dielectric constant of the breast tissue.

We have performed the simulation using the chest section of numerical whole-body voxel human model of Japanese adult female developed by National Institute of Information and Communications Technology (NICT), Kitasato University, Keio University, and Tokyo Metropolitan University [40]. The model is $160.0 \mathrm{~cm}$ in height and $53.0 \mathrm{~kg}$ in weight, which is completed by using the MRI images of human body. It was shown that the tumor of $5 \mathrm{~mm}$ in diameter located at 2-3 cm depth from the skin can be detected by using the model.

\subsubsection{Ultrashort-pulse reflectometer}

The schematic of a USPR is shown in Fig. 14. A probe beam is an $8 \mathrm{~V}, 65$ ps FWHM pulse. The FFT spectrum extends 8-10 GHz. The impulse is fed to a planar-type antenna. The reflected wave detected by an identical antenna is recorded by a high-speed sampling scope. The antennas attached to a rotational stage with a stepping motor are moved every 5 degree around the breast model from 0 to 180 degrees through the top. The reflected waves from the breast model are received at each antenna location, thus 37 reflected waves are provided. For the present experiment, we use a phantom model simulated for real breast biopsy (CRIS: model 051) as well as an oil model. The picture is shown in Fig. 14, which have a relative dielectric constant close to 20 and attenuation of $19 \mathrm{~dB} / \mathrm{cm}$ at $8 \mathrm{GHz}$. The phantom skin layer is $55-\mathrm{mm}$ higher, lying $20 \mathrm{~mm}$ below from the antenna elements. This distance between the antennas and the phantom provides a reasonable coverage of a model by an antenna radiation pattern. Dielectric materials with $6-9 \mathrm{~mm}$ in diameter used as tumors are located at around 10-25 mm depth from the top of the breast model.

The antennas should efficiently focus the microwave signal towards the target and collect the back-scattered power. A wideband antenna with unidirectional radiation pattern is required for these applications. Moreover, the antenna should be compact enough for easy installation, 
integration with other electronic circuits, and the ease in fabrication of the arrays. Microstrip antennas are compact, but they exhibit narrow bandwidth. This makes them unsuitable for timedomain applications. The Vivaldi antenna is selected since it provides wideband characteristic with acceptable performance as the present frequency response. In Fig. 15 is shown the measurement of S parameters and antenna gain as a function of frequency. We can see that the frequency band is from 1 to $10 \mathrm{GHz}$ which can be suitable for the present USPR experiment.

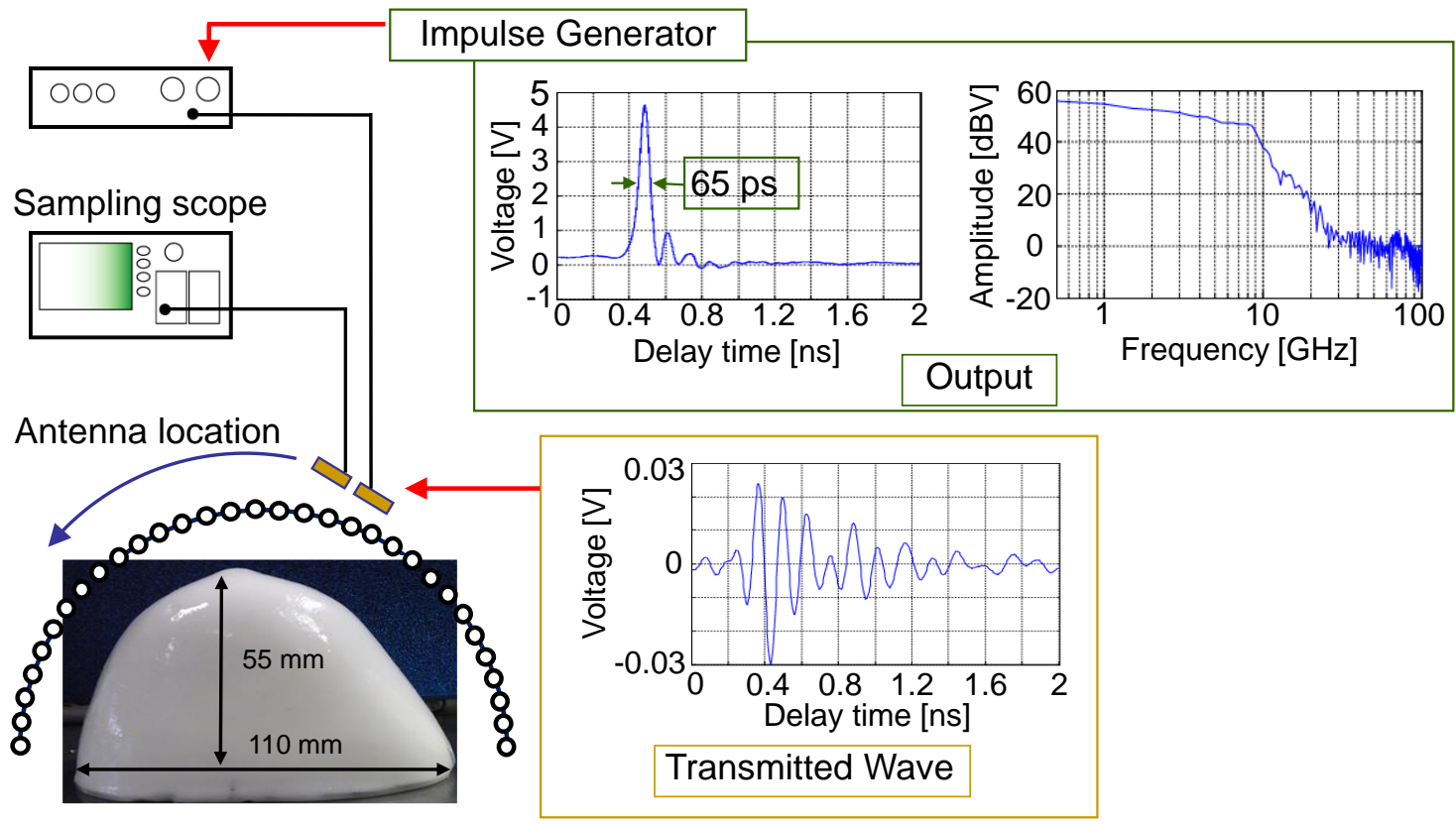

Fig. 14. Experimental apparatus of the USPR.
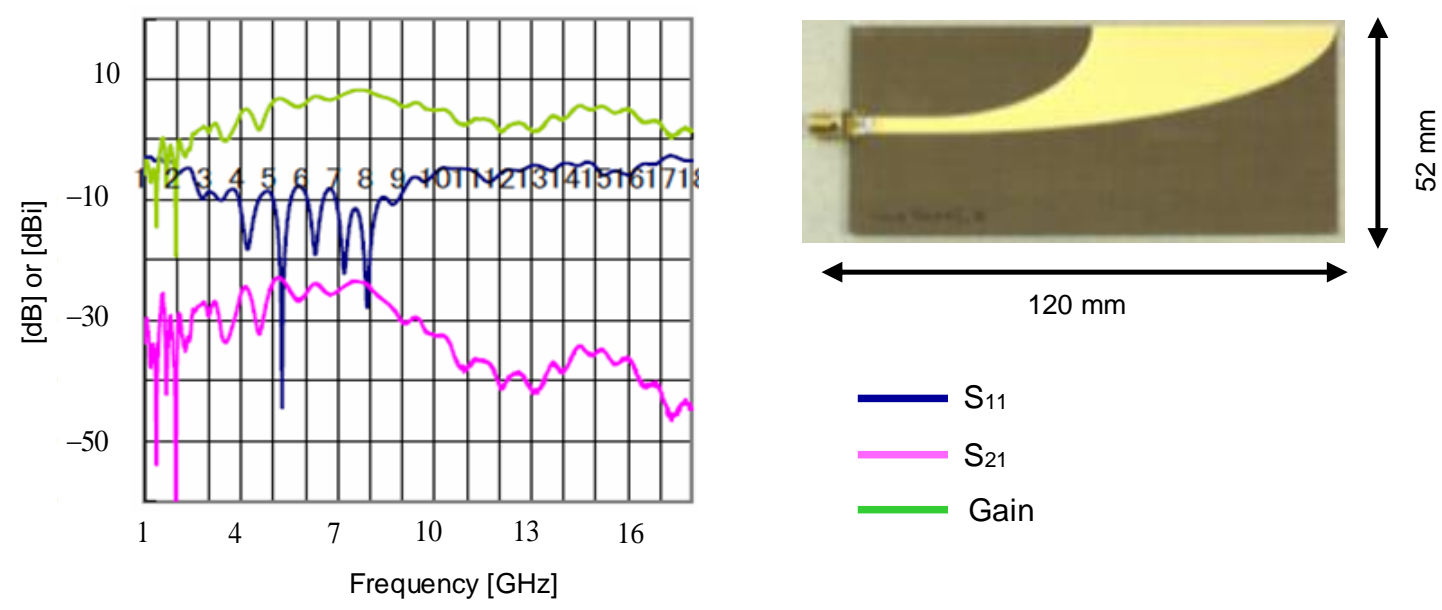

Fig. 15. Large size Vivaldi-antenna and its S parameters and gain. The Vivardi antenna has the same (up side down) configuration in the reverse side. 


\subsubsection{Experimental results}

Before we apply an image reconstruction, the tumor response must be extracted from the measurement data efficiently. The data contains additional undesired signals such as the antenna coupling, the reflections from the skin as well as the tumor response. Usually we can subtract all the unwanted signals by the measured signal without the tumor. This method is called background subtraction. It can be treated as the ideal way of extracting tumor response. However, it can not be used with real patients. We have applied three methods in order to extract the spurious signals.

The frequency region and bandwidth are selected to give the best reconstructed imaging by applying the following processes to the measurement signal, that is, FFT of the reflected wave, select the frequency range, IFFT of the selected components, and reconstruction. The tumor response is smaller in the high frequency region than that in the low frequency region. Considering the frequency band to affect the image reconstruction, we have selected some results in several frequency bandwidths from 2-7 GHz to $2-10 \mathrm{GHz}$, the results show very little variations. When we fix the frequency bandwidth as $6 \mathrm{GHz}$ but shift the start frequency from 2 to $5 \mathrm{GHz}$, the clutter increases and imaging become unclear for the start frequency of upper than $4 \mathrm{GHz}$.

The reflected waves from the surface of the breast model and the object can be seen in Fig. 16(a). The image reconstruction is performed after removing the ringing components from this remaining signal. Figure 16(b) shows an example of the reconstructed image for the frequency band of $1-10 \mathrm{GHz}$ with a permittivity of the targets is 20 .

(a)

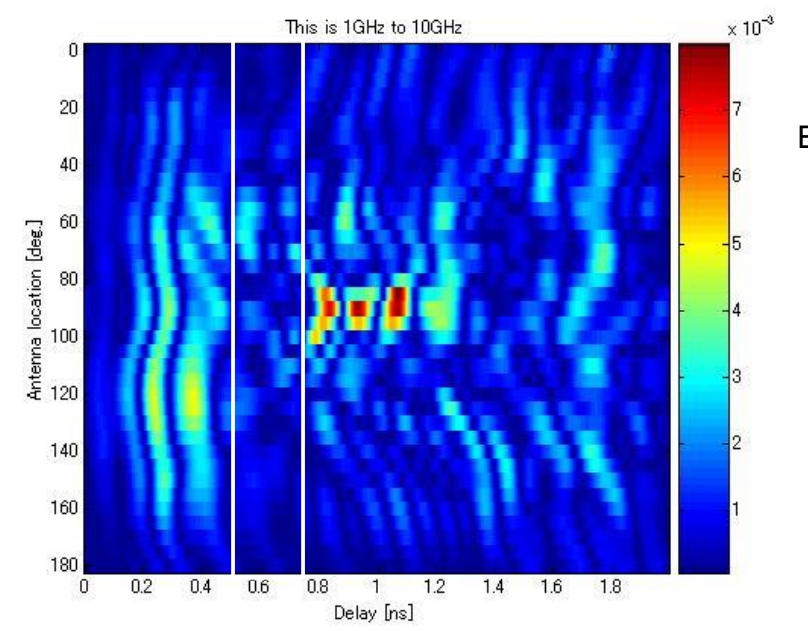

(b)
Enlarge figure $0.5 \sim 0.8 \mathrm{~ns}$
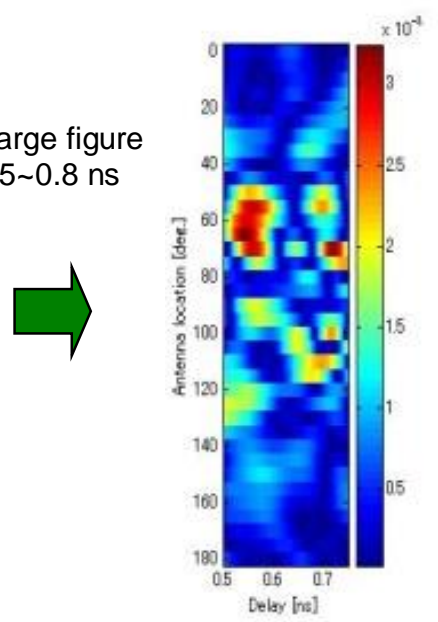

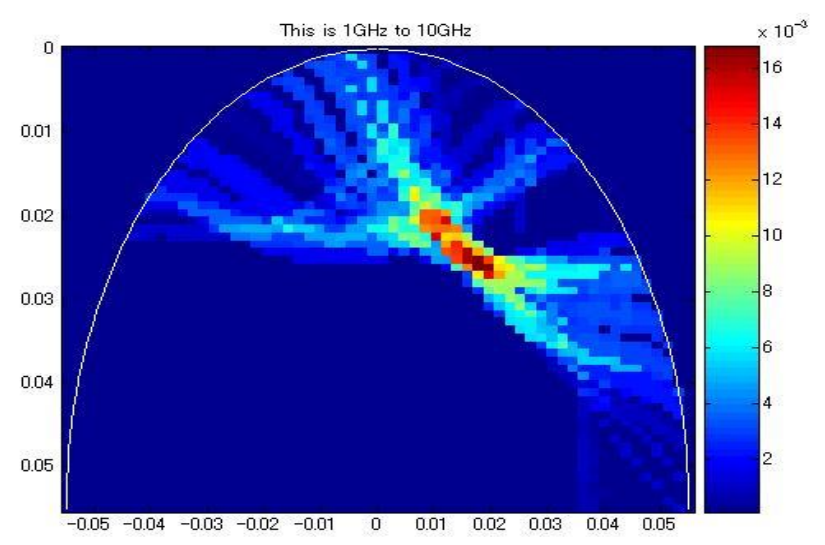


Fig. 16. Image reconstruction: (a) reflected waves at each position, (b) reconstruction of the object. The object's permittivity is $\varepsilon_{r}=20$.

\section{Conclusions}

Microwave reflectometry is a diagnostic tool that uses radar techniques. It has widely been used in magnetically confined plasmas, since it provides good spatial and temporal resolutions while requiring a single viewing chord. The combination of reflectometry and $1 \mathrm{D} / 2 \mathrm{D}$ detector array thus gives $2 \mathrm{D} / 3 \mathrm{D}$ profiles of plasma, which is called microwave imaging reflectometry (MIR). This MIR system has been applied to the LHD plasma. The feature of the system is the use of incident beam with 4 different frequencies and 2D detector array (horn mixer array). The MIR gives temporal and spatial characteristics of the MHD instability in LHD.

Microwave reflectometry has also been applied to biological measurements. We have proposed new signal processing techniques for microwave reflectometer sensing that enable estimation of mental stress from measurement of body dynamic motion. The algorithm based on the MEM is studied to reconfigure the HRV correctly and automatically from low-S/N microwave reflectometer signals. This technique enables the stress evaluation by microwave reflectometers in any environment, and indicates a possibility of the real-time evaluation of stress toward detection of stress syndrome or sleep prediction in the daily life, for example, while driving.

We have also performed the imaging diagnostics for breast cancer detection using a phantom model which is approximate to the actual breast tissue. It is effective to detect the breast cancer tumor by confocal microwave imaging (CMI) using background subtraction method to give better signal-to-noise ratio (SNR).

\section{Acknowledgments}

This work is partly supported by the Grant-in-Aid for Scientific Research, The Ministry of Eduaction. Science, Sports, and Culture (No. 16082205, No. 20360186 and No. 21246140), the Grant for Practical Application of University R\&D Results under the Matching Fund Method, NEDO, and by Adaptable and Seamless Technology Transfer Program through target-driven R $\&$ D, JST

\section{References}

[1] C. Laviron, A. J. H. Donné, M. E. Manso, and J. Sanchez, Reflectometry techniques for density profile measurements on fusion plasmas, Plasma Phys. Control. Fusion 38 (1996) 905.

[2] N. Bretz, Diagnostic instrumentation for microturbulence in tokamaks, Rev. Sci. Instrum. 68 (1997) 2927.

[3] H. J. Hartfuss, RF techniques in plasma diagnostics, Plasma Phys. Control. Fusion 40 (1998) A231.

[4] R. Nazikian, G. J. Kramer, and E. Valeo, A tutorial on the basic principles of microwave reflectometry applied to fluctuation measurements in fusion plasmas, Phys. Plasmas 8 (2001) 1840.

[5] G. D. Conway, J. Schirmer, S. Klenge, W. Suttrop, E. Holzhauer and the ASDEX Upgrade Team, Plasma rotation profile measurements using Doppler reflectometry, Plasma Phys. Control. Fusion 46 (2004) 951. 
[6] V. L. Ginzburg, The Propagation of Electromagnetic Waves in Plasmas (Pergamon, Oxford, 1970).

[7] L. G. Bruskin, A. Mase, T. Tamano, and K. Yatsu, Application of one-dimensional WentzelKramers-Brillouin approximation in microwave reflectometry of plasma density profiles, Rev. Sci. Instrum. 69 (1998) 2184.

[8] E. Mazzucatto, T. Munsat, H. Park, B. H. Deng, C. W. Domier, N. C. Luhmann, Jr., A. J. H. Donné, and M. van de Pol, Fluctuation measurements in tokamaks with microwave imaging reflectometry Phys. Plasmas 9 (2002) 1955.

[9] H. Park et al., Recent advancements in microwave imaging plasma diagnostics, Rev. Sci. Instrum. 74 (2003) 4239.

[10] M. Ignatenko, A. Mase, L. G. Bruskin, Y. Kogi, and H. Hojo, Numerical study of microwave imaging reflectometry for measurements of density fluztuataions in a tandem mirror plasma, Nucl. Fusion 46 (2006) S760.

[11] S. Yamaguchi, Y. Nagayama, D. Kuwahara, T. Yoshinaga, Z. B. Shi, Y. Kogi, and A. Mase, Development of microwave imaging reflectometry in Large Helical Device, Rev. Sci. Instrum. 79 (2008) 10F111.

[12] D. Kuwahara, S. Tsuji-Iio, Y. Nagayama, T. Yoshinaga, M. Sugito, Z. Shi, S. Yamaguchi, Y. Kogi, and A. Mase, Development of 2-D Antenna Array for Microwave Imaging Reflectometry in LHD, J. Plasma Fusion Res. SERIES 8 (2009) 649.

[13] T. Yoshinaga, Y. Nagayama et al., Simultaneous projection and detection system of four different frequencies for microwave imaging reflectometry in Large Helical Device, Rev. Sci. Instrum. 81 (2010) 10D915.

[14] Y. Kogi, T. Sakoda, A. Mase, N. Ito, Y. Yokota, S. Yamaguchi, Y. Nagayama, S. H. Jeong, M. Kwon, and K. Kawahata, Development of multi-channel IF system for ECE radiometer on KSTAR tokamak, Rev. Sci. Instrum. 79 (2008) 10F115.

[15] D. Kuwahara, S. Tsuji-Iio, Y. Nagayama, T. Yoshinaga, Z. Shi, S. Yamaguchi, M. Sugito, Y. Kogi, and A. Mase, Upgrade of 2-D Antenna Array for Microwave Imaging Reflectometry and ECE Imaging, J. Plasma Fusion Res. SERIES 9 (2010) 125

[16] T. Yoshinaga, D. Kuwahara, Y. Nagayama, H. Tsuchiya, S. Yamaguchi, Y. Kogi, S. Tsuji-Iio, H. Hojo, and A. Mase, Optics Design for Microwave Imaging Reflectometry in LHD, Plasma Fusionj Res. 5 (2010) 030.

[17] G. D. Conway and G. Vayakis, Specialist Working Group on Reflectometry, Report to ITPA-16 Plasma Diagnostics, St. Petersburg, Russia, April 20-24, 2008.

[18] Z. Shen, N. Ito, Y. Liang, L. Lin, C. W. Domier, M. Johnsaon, N. C. Luhmann, Jr., A. Mase, and E. Sakata, Protection Filters in ECEI Systems for Plasma Diagnostics, Plasma Fusion Res. 2 (2007) 1030 .

[19] C. C. Chen, Transmission of Microwave Through Perforated Flat Plates of Finite Thickness, IEEE Trans. Microw. Theory Tech. 21 (1973) 1.

[20] Y. Nagayama et al., Development of Microwave Imaging Diagnostics in LHD, Plasma Fusion Res. 87 (2011) 359 (in Japanese).

[21] J. C. Lin, Non-invasive microwave measurement of respiration, Proc. IEEE 63 (1975). 1530. 
[22] P. C. Pedersen, C. C. Johnson, C. H. Durney, and D. G. Bragg, An investigation of the use of microwave radiation for pulmonary diagnostics, IEEE Trans. Biomed. Eng. 23 (1976) 410.

[23] K. M. Chen, Y. Huang, J. Zhang, A. Norman, Microwave life-detection system for searching human subjects under earthquake rubble or behind barrier, IEEE Trans. Biomed. Eng. 27 (2000) 105.

[24] A. D. Droitcour, O. B. Lubecke et al., Range correlation and I/Q performance benefits in singlechip silicon doppler radars for noncontact cardiopulmonary monitoring, IEEE Trans. Microw. Theory Tech. 52 (2004) 838.

[25] L. Duvnjaek, S. Vuckovic, N. Car, and Z. Metelko, Relationship between autonomic function, 24-h blood pressure, and albuminuria in normotensive, normoalbuminuric patients with Type 1 diabetes, J. Diabetes Complications 15 (2001) 314.

[26] U. Wiklund, M. Akay, and U. Niklasson, Short-term analysis of heart-rate variability of adapted wavelet transforms, IEEE Eng. Med. Biol. Mag. 16 (1997) 113.

[27] M. Takada, T. Ebara, and Y. Sakaki, The Acceleration Plethysmography system as a new physiological technology for evaluating autonomic modulations, HEP. 35 (2008) 373.

[28] S. Suzuki, T. Matsui, H. Imuta, M. Uenoyama, H. Yura, M. Ishihara, and M. Kawakami, A novel autonomic activation measurement method for stress monitoring-non-contact measurement of heart ratevariability using a compact microwave radar, Med. Biol. Eng. Comput. 46 (2008) 709.

[29] D. Nagae and A. Mase, Measurement of heart rate variability and stress evaluation by using microwave reflectometric vital signal sensing, Rev. Sci. Instrum. 81 (2010) 094301; Virtual J. Biolog. Phys. Res. 20 (2010).

[30] J. P. Burg, Paper presented at the $37^{\text {th }}$ Ann. Int. Meeting, Soc. of Explor. Geophys., Oklahoma, Oct. 1967.

[31] See "http://cmm.cit.nih.gov/maxent/letsgo.html" for the MEM of data analysis.

[32] P. Burbaud, P. Degreze, P. Lafon, J. M. Franconi, B. Bouligand, B. Bioulac, J. M. Caille, and M. Allard, Lateralization of prefrontal activation during internal mental calculation: a functional magnetic resonance imaging study, J. Neurophysiol. 74 (1995) 2194.

[33] H. Mizuhara, Li-Q. Wang, K. Kobayashi, and Y. Yamaguchi, A long-range cortical network emerging with theta oscillation in a mental task, Neuroreport 15 (2004) 1233.

[34] M. Lazebnik, D. Popovic et al., A large-scale study of the ultrawideband microwave dielectric properties of normal, benign and malignant breast tissue obtained from cancer surgeries, Phys. Med. Biol. 52 (2007) 6093.

[35] H. Hojo, Y. Kurosawa, and A. Mase, Development of microwave diagnostic simulator, Rev. Sci. Instrum. 70 (1999) 983.

[36] S. C. Hagness, A. Taflove, and J. E. Bridges, Two-dimensional FDTD analysis of a pulsed microwave confocal system for breast cancer detection: fixed-focus and antenna-array sensors, IEEE Trans. Biomed. Eng. 45 (1998) 1470.

[37] $\mathrm{Xu} \mathrm{Li} \mathrm{and} \mathrm{S.} \mathrm{C.} \mathrm{Hagness,} \mathrm{Confocal} \mathrm{Microwave} \mathrm{Imaging} \mathrm{Algorithm} \mathrm{for} \mathrm{Breast} \mathrm{Cancer} \mathrm{Detection,}$ IEEE Microwave Wireless Comp. Lett. 11 (2001) 130.

[38] E. C. Fear, Xu Li, and S. C. Hagness, Confocal microwave imaging for breast cancer ditection: localization of tumors in three dimensions, IEEE Trans. Biomed. Eng. 49 (2002) 812. 
[39] M. Klemm, I. J. Craddock, J. A. Leendertz, A. Preece, and R. Benjamin, Radar-based breast cancer detection using a hemispherical antenna array-Experimental results, IEEE Trans. Antennas Propag. 57 (2009) 1692.

[40] T. Nagaoka, S. Watanabe et al., Development of realistic high-resolution whole-body voxel models of Japanese adult male and female of average height and weight, and application of models to radio-frequency electromagnetic-field dosimetry, Phys. Med. Biol. 49 (2004) 1. 